\title{
Valores y creencias en el proceso de modernización
}

\author{
Mercedes Fernández Alonso \\ Universidad de Málaga. Área de Sociología \\ mfalonso@uma.es
}

Recibido: 23-01-2008

Aceptado: 20-01-2009

\section{Resumen}

El objetivo de este trabajo es conocer el cambio de valores habido en la población andaluza en los últimos diez años y determinar, a partir de ahí, su grado de modernización. Para ello, se han seguido dos criterios conjuntamente: uno comparativo y otro teórico. El primero, una comparación de los datos de Andalucía con los de Cataluña. Por su parte, el criterio teórico se basa en la teoría de la modernización desarrollada por Inglehart y Welzel (2006). Los resultados del trabajo confirman la hipótesis de partida: la distancia entre ambas comunidades aumenta con el cambio de valores ocurrido en los últimos años, tanto en la proporción de materialistas como en la de post-materialistas. Otra cuestión importante es el reciente repunte en Andalucía de valores materialistas o asociados a la seguridad económica y personal.

Palabras clave: valores, materialismo, post-materialismo, modernización.

Abstract. Values and belief system in the modernisation process

This article explores continuity and change of values in the Andalusian population during the last ten years, thus attempting to explain the degree of modernization in this region. To achieve this aim, two methods have been followed: one comparative and another one theoretical. A comparison of data in Andalusia and in Cataluña is underscored by the work on modernisation by Inglehart and Welzel (2006). The results of this study affirm the initial hypothesis: detachment in relation to materialism and post-materialism in both communities increased over the last ten years. It is important too the recent growth in Andalusia of materialistic values or values associated to the economic and personal security.

Key words: values, materialism, post-materialism, modernisation.

\section{Sumario}

Introducción Consideraciones finales.

Marco teórico general El postmaterialismo y los cambios sociales

Método. Definición operativa de materialismo y postmaterialismo acaecidos en los últimos años

Conclusiones

Hallazgos y discusión Referencias bibliográficas 


\section{Introducción}

Este trabajo analiza el cambio de valores que se ha producido en la población andaluza en los últimos diez años para determinar su grado de modernización. La perspectiva teórica tomada como referencia es la teoría de la modernización desarrollada por Inglehart y Welzel (2006) ${ }^{1}$, que defiende que el cambio social implica el paso de los valores materialistas a los postmaterialistas. Se ha realizado un análisis comparado entre la comunidad autónoma andaluza y la catalana. La hipótesis de partida establece que Cataluña es una región más moderna (desarrollada) - y, por tanto, con mayor presencia de valores postmaterialistas- y Andalucía sigue sus pasos. Respecto al marco temporal, se han tenido en cuenta dos momentos distintos: los años 1995 y 2006.

\section{Marco teórico general ${ }^{2}$}

El análisis de los valores es uno de los campos más fructíferos en la actualidad dentro del marco teórico de la modernidad (Inglehart y Welzel, 2006; Welzel, Inglehart y Klingemann, 2003; Del Pino y Bericat, 1998; Orizo, 1996; Carnero, 1992; Inglehart, 1991; Eisenstadt, 1964). Los valores relacionados con el progreso y el crecimiento son una importante base de apoyo en los procesos de desarrollo. Para que una sociedad alcance un determinado grado de modernización y bienestar, necesita una voluntad social de desarrollo. Los hábitos, las costumbres o las actitudes de la población también influyen en el comportamiento económico y en la capacidad de crecimiento ${ }^{3}$. Por lo tanto, en el proceso dinámico de cambio social, cultura y desarrollo se ven inmersos en un proceso recíproco. Esto es, la influencia de una variable sobre la otra se produce en ambos sentidos: las instituciones moldean a la cultura (argumento marxista ${ }^{4}$ y los valores culturales moldean a las instituciones (argumento weberiano ${ }^{5}$ ) (Requena, 2002: 1027 y 1028).

Sin embargo, como señala Inglehart (1991), los factores culturales han sido escasamente explorados y medidos cuantitativamente. A pesar de ello,

1. El proceso de modernización en esta investigación se analiza desde la perspectiva de estos autores. No obstante, cabe mencionar otras perspectivas, por ejemplo: las teorías clásicas de la modernización (Comte y Spencer, Marx y Weber, Durkheim y Tönnies), las teorías de la convergencia y las de la transición postcomunista. Para un análisis más detallado de las mismas, véase Sztompka (1995: 155-167) o Solé (1976).

2. Este trabajo forma parte de otro más amplio subvencionado por el Centro de Estudios Andaluces (Ref. SOCH1.07/023), entidad dependiente de la Consejería de la Presidencia de la Junta de Andalucía.

3. Como diría Max Weber en su obra La ética protestante y el espiritu del capitalismo:, "[en tales casos] no era la afluencia de dinero nuevo lo que provocaba esta revolución, sino el nuevo espíritu, el "espíritu del capitalismo" que se había introducido [...] Hombres educados en la escuela de la vida, prudentes y arriesgados a la vez, sobrios y perseverantes, entregados, de lleno y con devoción a lo suyo, con concepciones y "principios" rígidamente burgueses" (Weber, 1979: 68-70).

4. Sobre este tema, véase Marx (1986).

5. Véase Weber (1979). 
representan un componente fundamental de cualquier sistema social. Las grandes transformaciones que se han dado en la sociedad en los últimos años han venido acompañadas por una serie de mutaciones en los valores de los individuos. La investigación sociológica sobre esta temática de los valores, articulada en los últimos años sobre los trabajos de la World Values Survey y la European Values Survey, sostiene la teoría general de la transición desde los valores materialistas hasta los postmaterialistas o, en términos más actuales (Inglehart y Welzel, 2006), desde los valores de escasez o supervivencia hasta los valores de autoexpresión, desde los valores de dependencia hasta los valores de emancipación. Cuanto más moderna y desarrollada sea una sociedad, más éxito y divulgación tendrán los valores postmaterialistas.

Esa tendencia no puede hacernos olvidar la permanencia de elementos culturales tradicionales en las sociedades desarrolladas. Partiendo de la tesis de Inglehart y Welzel (2006: 26), al hablar de valores y creencias en momentos de cambios políticos y económicos, nos enfrentamos a dos ideas más complementarias que contradictorias: en primer lugar, la permanencia de las tradiciones en nuestras vidas y, en segundo lugar, por el contrario, una industrialización que promueve la decadencia de valores tradicionales y su sustitución por otros modernos ${ }^{6}$.

En lo que a la primera suposición se refiere, se parte de la premisa de la persistencia de las tradiciones culturales independientemente de las condiciones políticas y económicas de las sociedades. Por tanto, los valores se asumen como relativamente independientes de las condiciones económicas, esto es, la influencia de las tradiciones culturales no desaparece. Por otro lado, y en lo referente a la segunda suposición, parece significativo que el desarrollo socioeconómico ocasiona, a largo plazo, cambios predecibles (véase Turner, 1971). Los teóricos de la modernización (Marx, Bell o Toffler, entre otros) relacionan el surgimiento de la sociedad industrial con movimientos culturales que se apartan de los sistemas de valores tradicionales. En definitiva, un declive de estos y su sustitución por otros modernos ${ }^{7}$.

6. En esta línea, tendría cabida la «hipótesis de la bifurcación posmoderna» a la que hace referencia Eduardo Bericat. En la postmodernidad, aparte de caminar hacia la hipermodernización, también están presentes procesos de «tradicionalización»: el individuo postmoderno demanda más modernidad y, al mismo tiempo, más tradición. En sus propias palabras: «lo que pretende poner de manifiesto es que el modo de vida tradicional surgió de la aplicación de unos recursos, potencialidades o virtualidades humanos, de carácter universal e intemporal, a un contexto específico, y que estos recursos, potencialidades y virtualidades, que fueron en gran parte reprimidos por la sociedad moderna, serán re-activados, re-adaptados y re-actualizados en un nuevo contexto de hipermodernización» (Bericat, 2003: 38).

7. La teoría de la modernización (Inglehart y Welzel, 2006: 22) nace en la era de la Ilustración bajo el ideal de controlar la naturaleza mediante la razón. Más explícitamente, Antoine de Condorcet destaca por ser uno de los autores que primero vincula claramente desarrollo económico y cambio cultural. Para él, el progreso tecnológico y el desarrollo económico llevarán de manera inevitable a transformaciones en los valores de las personas. 
Para Inglehart y Welzel (2006: 27-35), el eje central de la teoría de la modernización de los valores (el paso del materialismo al postmaterialismo) es que el desarrollo socioeconómico se encuentra relacionado con los cambios que se han producido, y ello empuja a las sociedades en una dirección predecible, eso sí, no determinista ${ }^{8}$. Dicho cambio se origina con la innovación tecnológica, que aviva la productividad laboral, lo que produce especialización, aumento de los niveles educativos y de renta, y diversificación de la interacción humana (efecto de individuación ${ }^{9}$ y liberación), con lo cual se enaltecen las relaciones de la negociación.

Conforme se avanza en el tiempo, esta situación acaba favoreciendo que se den cambios culturales en los fenómenos sociales, entre ellos, en los roles de género, en las actitudes hacia la autoridad, en el aumento de la participación política, etc. Todo ello se aprecia, a modo de resumen, en el hecho que los seres humanos se transforman en un público difícilmente manipulable. Por lo tanto, cuando los miembros de una sociedad han experimentado elevadas cotas de prosperidad económica durante largos periodos de tiempo, se produce un cambio de valores. Todo ello recibe el nombre de modernización.

Más específicamente, para estos autores la influencia del desarrollo socioeconómico en el cambio cultural opera en dos fases. En un primer momento, con el traslado de la fuerza de trabajo del sector primario al secundario (la industrialización), los sujetos tienden a cambiar su visión del mundo y a sustituir el énfasis en los valores tradicionales por el énfasis en los seculares racionales. En un segundo momento, con el paso de los trabajadores del sector secundario al terciario o de servicios (sociedad postindustrial), se traslada la importancia de los valores de la supervivencia a la importancia de los valores de la autoexpresión (énfasis en la autonomía individual).

Según Bericat (2003: 33), Inglehart inició su investigación para demostrar que en las sociedades avanzadas se estaba produciendo un profundo cambio cultural basado en la creciente sustitución de los valores «materialistas» por los "postmaterialistas». Maslow, en su pirámide de las necesidades humanas, mantuvo la tesis de que las «necesidades materiales» (el mantenimiento y la seguridad física) estaban perdiendo prioridad valorativa respecto a las «necesidades no fisiológicas» (estima, pertenencia al grupo, autoexpresión, satisfacción intelectual, etc.). Para explicar este cambio, se basó en dos hipótesis fundamentales: en primer lugar, la "hipótesis de la escasez», es decir, otorgar mayor

8. Como recuerda Díez Nicolás (2007), el propio Inglehart ha subrayado que no todas las sociedades se mueven al mismo ritmo. De hecho, el cambio lineal hacia los valores de autoexpresión y hacia valores seculares y racionales también ha experimentado «vueltas atrás» (véase el caso de los países excomunistas y de algunas sociedades menos desarrolladas).

9. Gobernado (1996: 9) considera proceso de modernización al "progresivo aumento de la conciencia del "yo" frente a la conciencia del "nosotros", a la creciente individuación de la sociedad [...] se convierten en indicadores de modernización la aleatoriedad en el comportamiento; la independencia del comportamiento individual con respecto a las categorías sociales que obren como variable independiente, así como las posibilidades de movilidad social y de movilidad espacial». 
valor subjetivo a las cosas ciertamente escasas, por lo que, en una sociedad de la abundancia (por ejemplo, con el surgimiento del Estado del bienestar que ha asegurado la existencia material), se valorará mayoritariamente la satisfacción de las necesidades no fisiológicas. A continuación, la «hipótesis de la socialización», esto es, los valores no se ajustan inmediatamente a nuevos contextos, sino que necesitan de un proceso de socialización para adaptarse a las nuevas generaciones.

Para Requena (2004: 336), este cambio de dirección en los procesos de cambio refleja el "principio de utilidad marginal decreciente». Las sociedades avanzadas han visto modificada su trayectoria por diversos factores, entre ellos, ha cambiado el sistema de valores. El acento en el logro económico individual fue uno de los principales elementos que favoreció la modernización. Sin embargo, una vez cubiertas las necesidades materiales de supervivencia, el sistema de valores se enfoca a la consecución de otras metas, fundamentalmente las relacionadas con la calidad de vida. Esto es, la posibilidad de elegir qué estilo de vida queremos vivir y cómo queremos autoexpresarnos. Se ha dado el paso de los valores materialistas (modernos), que subrayan la seguridad física y económica, a los postmaterialistas (postmodernos), que recalcan la autoexpresión y la calidad de vida.

En definitiva, el desarrollo socioeconómico aumenta considerablemente los niveles de seguridad existencial, por lo que contribuye al desarrollo humano. A lo largo de este proceso, las personas se sienten liberadas de las dependencias materiales en sus elecciones diarias. Así se explica el incremento de los valores de la autoexpresión, lo cual facilita a las personas ir más allá de la pura supervivencia. En otras palabras, la modernización es un aspecto del desarrollo humano. La amplia amalgama de cambios socioeconómicos lleva implícitas transformaciones culturales que favorecen el surgimiento de los valores de autoexpresión o de emancipación (libertad de expresión y de elección, autonomía individual, igualdad y participación) que favorecen una sociedad o una cultura humanista, es decir, creativa y centrada en las personas.

A largo plazo, esta cultura humanista se extiende por los diversos dominios de la vida y ello contribuye a promover cambios en las distintas esferas sociales. Ahora es cuestión de elección individual. Los nuevos valores están remodelando, entre otros factores, la religiosidad o las creencias religiosas; los roles de género; los valores familiares; las motivaciones o las actitudes laborales; los valores o las actitudes tecnocientíficos; las actividades comunitarias; las relaciones entre las personas, la naturaleza o el entorno, y la participación política.

En la modernización, dice Díez Nicolás en la introducción al libro de Inglehart y Welzel (2006): «[...] se demuestra una vez más que el eje que mide el cambio desde los "valores materialistas" a los "valores postmaterialistas", desde los "valores de escasez o de supervivencia" a los "valores de autoexpresión", desde los "valores de dependencia o sumisión" a los "valores de emancipación" es mucho más útil como variable explicativo-predictiva que el otro eje, el del cambio desde los "valores tradicionales" a los "valores secular-racionales”» (Díez Nicolás, 2006: X). 
No obstante, creo conveniente hacer una puntualización al respecto. Según afirma recientemente Díez Nicolás (2007), en las últimas oleadas de la World Values Survey (WVS) se muestra una clara tendencia a la bajada del índice de postmaterialismo. Los valores de la autoexpresión han aumentado desde 1981 hasta 2005 en países como Argentina, Chile, Finlandia, Francia, Italia, Japón, Países Bajos, Eslovenia, España o EEUU, pero, debido al incremento de la actual inseguridad personal y económica (terrorismo internacional, bandas criminales procedentes de diferentes países o rápido incremento de la cantidad de inmigrantes, entre otros elementos), estos valores han empezado a descender en los últimos tiempos.

Como ya se indicó, el objeto general de la investigación consiste en establecer una comparación entre dos realidades sociales españolas: Andalucía y Cataluña. Más concretamente, el tema que nos ocupa verdaderamente es determinar el nivel de modernización de la sociedad andaluza en el ámbito de los valores, y la mejor manera de analizarlo es comparándola con otra sociedad. En este caso, la comparación es doble: por un lado, con Cataluña, por otro, con la sociedad existente hace unos años. Ésta es la mejor forma de acceder al análisis del cambio social. Como modelo teórico principal, siguiendo la tesis de Inglehart, el eje que mejor explica la modernización de una sociedad es el tránsito de los valores materialistas a los postmaterialistas, de los valores de la escasez a los de la autoexpresión, de los valores de la dependencia a los de la emancipación. Tal y como se dijo antes.

\section{Método. Definición operativa de materialismo y postmaterialismo}

Como base de datos sobre la extensión del materialismo y del postmaterialismo, se han utilizado los sondeos que realiza mensualmente la empresa $\mathrm{ASEP}^{10}$. Las encuestas se realizan sobre una muestra representativa de la población española de más de dieciocho años (1.200 entrevistas) estratificada por comunidades autónomas y por municipios según su tamaño. Para $p=q=50$ por 100 y un coeficiente de fiabilidad del 95,5 por 100 , el error muestral es de $\pm 2,89$. Los entrevistados se han seleccionado por rutas aleatorias y cuotas de sexo y edad. Todos los sondeos tienen un bloque de preguntas comunes, por lo que es posible la suma de los resultados, en estos casos, de las continuadas encuestas. Para los dos años que se van a analizar (1995 y 2006), la muestra de Andalucía asciende a 2.385 y a 2.297 casos, respectivamente, y la de Cataluña, a 2.094 y a 2.123 casos. También se han consultado fuentes de datos adicionales, principalmente la Encuesta de calidad de vida en el trabajo (1999 y 2004), elaborada por el Ministerio de Trabajo y Seguridad Social ${ }^{11}$.

10. Estas siglas significan Análisis Sociológicos, Económicos y Políticos SA, sociedad fundada por Juan Díez Nicolás en el año 1982.

11. La muestra de cada encuesta está formada nada más que por población ocupada en España y asciende a 6.000 personas entrevistadas. 
En ASEP, el índice de postmaterialismo es el porcentaje de entrevistados que tiene cinco puntos en la escala de postmaterialismo. Se obtiene a partir de la combinación de dos preguntas y sobre los objetivos postmaterialistas más importantes que deberían realizarse en España en los próximos diez años. Así, los entrevistados que mencionen "Dar a la gente más posibilidades para que participen en las decisiones importantes gubernamentales o políticas», «Proteger la libertad de expresión» (valores 2 o 4 ) y «Dar a la gente mayores facilidades para que participen en las decisiones importantes que tengan que ver con su trabajo o la comunidad en la que viven», "Proteger el medio ambiente», «Avanzar hacia una sociedad menos impersonal y más humana» $\mathrm{y}$ "Avanzar hacia una sociedad en la que las ideas sean más importantes que el dinero" (valores 3, 4, $7 \mathrm{u} 8$ ), se les asigna un punto. Por lo que dicho índice varía de 0 a 5 puntos (de menor a mayor grado de postmaterialismo) ${ }^{12}$.

Las variables empleadas en este estudio son las siguientes: sexo (categorías: «varón», «mujer»), edad (agrupada en cuatro categorías: «jóvenes» o de 18 a 24 años, «adultos» o de 35 a 44 años, «mayores» o de 45 a 64 años y «viejos» o de 65 y más años), nivel educativo (recodificado en "no sabe leer», «sabe leer», "primarios completos», «EGB o EPO», «FP o módulos», «BUP o ESO», «COU o bachiller», "Grado medio» y «Universitarios»), hábitat (clasificado en «rural» o menos de 10.000 habitantes, «semiurbano» o de 10.000 a 100.000 habitantes y «urbano» o más de 100.000 habitantes), índice de posición social de Galtung (elaborado teniendo en cuenta el sexo, la edad, la ocupación, la provincia de residencia, el hábitat de residencia y el nivel de ingresos en el hogar), estatus ocupacional (clasificado en «alto», "medio», «bajo», "en paro», «ama de casa», «jubilado», «estudiante» y «otros») e ideología política (con las categorías de «extrema izquierda», «izquierda», «centro izquierda», «centro», «centro derecha», «derecha» y «extrema derecha»).

\section{Hallazgos y discusión}

De los datos de la tabla $1^{13}$ se desprende que la población postmaterialista es, en general, minoritaria en ambas comunidades autónomas. La población de este tipo convive con población definida como materialista y con abundante población de tipo mixto o intermedio. No obstante, Cataluña acumula más población postmaterialista que Andalucía en los dos años considerados, como era de esperar según el modelo teórico. Además, y también de acuerdo con el

12. www.jdsurvey.net/jds/jdsurveyAnalisis.jsp

13. En la Encuesta de calidad de vida en el trabajo se da dos posibilidades de elección al entrevistado (en primera opción y en segunda opción). Ahora sólo se utilizará la primera opción, por facilitar la tarea. Cada respuesta es catalogada como materialista o postmaterialista fácilmente. Sumando los resultados, se obtiene una escala simple aditiva que cataloga la muestra entrevistada entre los dos extremos $(1=$ materialistas y 7 = postmaterialistas $)$. A partir de tal clasificación, se establecen tres categorías de población: la postmaterialista (valores 6 y 7 ), la materialista (valores 1 y 2 ) y una categoría intermedia (valores 3,4 y 5), que se denominará «mixtos». 
Tabla 1. Población materialista y postmaterialista en porcentajes por filas, según comunidad autónoma y año

\begin{tabular}{lllll}
\hline & Materialistas & Mixtos & Postmaterialistas & Total (N) \\
\hline 1999 & & & & \\
Andalucía & 23,4 & 67,4 & 9,2 & $100(892)$ \\
Cataluña & 15,1 & 71,9 & 13 & $100(1064)$ \\
\hline 2004 & & & & \\
Andalucía & 25,8 & 70,7 & 3,5 & $100(518)$ \\
Cataluña & 11,7 & 73,2 & 15,1 & $100(639)$ \\
\hline
\end{tabular}

Fuente: Encuesta de calidad de vida en el trabajo, 1999 y 2004. Elaboración propia.

modelo teórico, en Cataluña ha aumentado la proporción de población postmaterialista de un año a otro. Esto resulta lógico a juzgar por el incremento de PIB de esta comunidad autónoma ${ }^{14}$.

Sin embargo, se aprecia un dato discordante: en Andalucía hay menos población postmaterialista en el año 2004 que en el 1999. Pese al crecimiento correspondiente del PIB andaluz, muy notable también ${ }^{15}$, se ha producido un repunte de la población materialista o, lo que es igual, una disminución de la población postmaterialista.

La información anterior se encuentra apoyada también por los datos de ASEP. Esta organización clasifica la escala de postmaterialismo en cinco categorías. En el análisis que sigue, se han acumulado las dos últimas para formar la categoría de postmaterialista. Además, los datos se refieren a los años 1995 y 2006. Pese a estas diferencias, las tendencias en los resultados son las mismas: se aprecia una disminución evidente de la población postmaterialista en Andalucía, desde el año 1995 (9\%) hasta el 2006 (5,9\%). Por el contrario, en Cataluña se aprecia un leve aumento de tal población en la línea de lo previsto: desde el año 1995 (11,3\%) hasta el 2006 (14,2\%).

Posiblemente haya que reformular la tesis del postmaterialismo en momentos de gran repunte del PIB, o, como dice Díez Nicolás (2007), el incremento producido en los últimos años en los niveles de inseguridad económica y social, al que se hacía referencia en el apartado anterior, explicaría el aumento de los valores materialistas. De cualquier forma, siguiendo nuestros objetivos, la distancia entre Andalucía y Cataluña aumenta con el cambio de valores ocurrido en los últimos años: la distancia aumenta tanto en la proporción de materialistas, en este caso a favor de Andalucía, como en la de postmaterialistas, donde Cataluña se adelanta a la citada comunidad.

14. Según el Instituto de Estadística de Andalucía, el PIB (a precios de mercado) en Cataluña ha pasado de 82.752.572 en 1995 a 182.735 .639 en 2006 (en miles de euros).

15. Según el Instituto de Estadística de Andalucía, el PIB (a precios de mercado) en Andalucía ha pasado de 58.703.745 en 1995 a 135.294 .679 en 2006 (en miles de euros). 
A continuación, se va a ver cómo influyen o la relación que existe entre algunas variables en la escala de valores de la población. Concretamente, el sexo, la edad, el hábitat, el nivel educativo, la posición social (índice de posición social de Galtung ${ }^{16}$ ), la situación laboral u ocupación y la ideología política. Para ello, vamos a centrarnos ahora más específicamente en la escala de postmaterialismo empleada por ASEP. Como se ha indicado anteriormente, se encuentra formada por cinco categorías, pero, por razones de manejabilidad de la amplia amalgama de datos ${ }^{19}$, se van a reflejar, como se puede ver en la tabla 2 , los extremos ( 1 y 5 , este último representaría a los "postmaterialistas puros») y la categoría intermedia (3).

En primer lugar, no se encuentra una relación significativa entre postmaterialismo y género. Se aprecia, en general y en el intervalo de tiempo analizado (años 1995 y 2006), una situación similar en ambas comunidades autónomas: escasas diferencias por género y un pequeño despunte de varones con niveles de postmaterialismo más altos ${ }^{18}$.

En el caso de la edad, sí se observa una fuerte relación. Se cumple en ambas realidades la misma tendencia: a menor edad, mayor presencia de actitudes postmaterialistas y viceversa. Se trata sobre todo de un cambio generacional, es decir, resulta lógico que los más jóvenes se muestren más postmaterialistas, si atendemos al proceso de socialización de las nuevas generaciones. En los prácticamente últimos cincuenta años, nuestro país ha sufrido grandes transformaciones sociales que han afectado a todos los niveles de la vida. De la misma manera, los valores han ido cambiando, y así se transmiten a los individuos en el seno de las familias desde sus primeros años de vida (véase Comas, 2003). La estabilidad y el crecimiento económico de los países más desarrollados han sido interiorizados especialmente y, más fácilmente, por las generaciones más jóvenes que no han vivido épocas anteriores, no poseen hábitos o visiones fuertemente arraigadas y han experimentado más seguridad que los mayores. Así se explica su inquietud por la defensa del medio ambiente, de la libertad, de la participación o de la igualdad entre géneros, entre otros elementos.

Sin embargo, al comparar ambas comunidades, los jóvenes andaluces son más materialistas que los catalanes. Esta diferencia incluso se acrecienta con el paso del tiempo (un 22,6\% de jóvenes posicionados en el valor 1 de la escala

16. Elaborado teniendo en cuenta las siguientes variables: el sexo, la edad, la ocupación, la provincia de residencia, el hábitat y el nivel de ingresos en el hogar.

17. No obstante, en el análisis y en la interpretación de los resultados, se ha tomado en consideración el conjunto de la escala.

18. Estos resultados son corroborados por los del estudio Valores sociales en la cultura andaluza (del Pino y Bericat, 1998), aunque, por el contrario y en un principio, distan de los reflejados, por ejemplo, por la Encuesta de calidad de vida en el trabajo. Atendiendo a esta última fuente, en el primer año analizado (1999), especialmente en Andalucía, los hombres se muestran, a diferencia de lo comentado anteriormente, más materialistas y las mujeres, postmaterialistas. Sin embargo, con el paso de los años, las diferencias se acortan significativamente (por ejemplo, en el 2004 encontramos un 3,5\% de varones postmaterislistas frente a un $3,4 \%$ de mujeres postmaterislistas, proporción prácticamente idéntica). 
Tabla 2. Población postmaterialista según género, edad y hábitat, según comunidad autónoma y año

\begin{tabular}{|c|c|c|c|}
\hline 1 & 3 & $\begin{array}{l}\text { 5. Postmat. } \\
\text { Puros }\end{array}$ & Total \\
\hline And. Cat. & And. Cat. & And. Cat. & And. \\
\hline
\end{tabular}

\section{Género}

\begin{tabular}{cllllllll}
1995 Varón & 30,5 & 23,9 & 22,3 & 25,0 & 2,0 & 1,8 & $100(1.010)$ & $100(983)$ \\
Mujer (1) & 33,6 & 25,7 & 22,2 & 25,3 & 1,7 & 0,8 & $100(1.025)$ & $100(1009)$ \\
2006 Varón & 23,9 & 14,4 & 25,0 & 24,7 & 1,8 & 1,5 & $100(983)$ & $100(977)$ \\
Mujer (2) & 25,7 & 16,7 & 25,3 & 23,8 & 0,8 & 1,0 & $100(1.009)$ & $100(1.087)$ \\
\hline
\end{tabular}

\section{Edad}

\begin{tabular}{crrrlllll}
1995 Jóvenes & 22,6 & 13,9 & 29,2 & 32,3 & 3,2 & 4 & $100(877)$ & $100(718)$ \\
Adultos & 33,2 & 20,9 & 22,0 & 32,3 & 1,6 & 2,4 & $100(322)$ & $100(316)$ \\
Mayores & 37,1 & 28,4 & 17,0 & 24,6 & 0,7 & 1 & $100(560)$ & $100(589)$ \\
Viejos (3) & 50,4 & 35,8 & 11,2 & 21,0 & 0 & 0,4 & $100(276)$ & $100(324)$ \\
2006 Jóvenes & 21,5 & 8,5 & 27,2 & 27,2 & 1,3 & 2,1 & $100(717)$ & $100(662)$ \\
Adultos & 29,0 & 11,3 & 23,5 & 28,4 & 0,7 & 1,3 & $100(404)$ & $100(398)$ \\
Mayores & 21,8 & 17,0 & 27,4 & 24,7 & 0,9 & 0,9 & $100(537)$ & $100(575)$ \\
Viejos (4) & 31,7 & 28,9 & 19,2 & 15,2 & 2,7 & 0,5 & $100(334)$ & $100(429)$ \\
\hline
\end{tabular}

\section{Hábitat}

$\begin{array}{ccccccccc}1995 \text { Rural } & 35,3 & 22,6 & 23,5 & 28,3 & 1,0 & 1,8 & 100(502) & 100(508) \\ \text { Semiurbana } & 32,1 & 29,5 & 20,8 & 23,8 & 1,2 & 0,8 & 100(742) & 100(713) \\ \text { Urbana (5) } & 30,0 & 21,9 & 22,9 & 24,3 & 2,9 & 1,4 & 100(791) & 100(771) \\ 2006 \text { Rural } & 22,6 & 12,2 & 28,3 & 24,7 & 1,8 & 1,3 & 100(508) & 100(304) \\ \text { Semiurbana } & 29,5 & 16,9 & 23,8 & 25,2 & 0,8 & 1,1 & 100(713) & 100(794) \\ \text { Urbana (6) } & 21,9 & 15,7 & 24,3 & 23,3 & 1,4 & 1,3 & 100(771) & 100(966)\end{array}$

\begin{tabular}{|c|c|c|c|c|c|c|c|c|c|}
\hline & Chi-cuad. & DF & Sig. & Coef. conting. & & Chi-cuad. & DF & Sig. & Coef. cor \\
\hline (1) Andalucía & 9.742 & 4 & 0,045 & 0,067 & Cataluña & 5.065 & 4 & 0,281 & 0,05 \\
\hline (2) & 5.065 & 4 & 0,281 & 0,050 & & 3.346 & 4 & 0,502 & 0,040 \\
\hline (3) & 150.409 & 12 & 0,000 & 0,262 & & 154.017 & 12 & 0,000 & 0,271 \\
\hline (4) & 31.456 & 12 & 0,002 & 0,125 & & 194.851 & 12 & 0,000 & 0,294 \\
\hline (5) & 25.871 & 8 & 0,001 & 0,112 & & 21.575 & 8 & 0,006 & 0,104 \\
\hline (6) & 21.575 & 8 & 0,006 & 0,104 & & 9.514 & 8 & 0,301 & 0,06 \\
\hline
\end{tabular}

Fuente: ASEP, 1995-2006. Elaboración propia.

en Andalucía y un 13,9\% en Cataluña en 1995, y un 21,5\% frente a un 8,5\%, respectivamente, once años más tarde). Esta situación puede estar relacionada con diferencias en la socialización o con las elevadas tasas de desempleo que caracterizan al mercado de trabajo andaluz. Esto promueve, como diría Orizo (1996: XLV): "una actitud de defensa, un firme asentamiento de los pies en el suelo y la atención a lo inmediato, todos ellos valores opuestos a actitudes tendentes al postmaterialismo». 
Contrariamente, conforme aumenta la edad, aumenta el grado de materialismo. En este caso, también sobresale Andalucía; por ejemplo: en 1995, la mitad $(50,4 \%)$ de los individuos de mayor edad se situaba en el primer valor de la escala frente a un $35,9 \%$ de catalanes ${ }^{19}$. Se vuelven a ver diferencias por comunidades.

Diez años más tarde, se observa un descenso de población posicionada en las situaciones extremas. En el caso de Andalucía, aumenta la población de tipo «mixto» y desciende, aunque por detrás de Cataluña, la población materialista en todos los grupos de edad (reflejo de un mayor grado de modernización que en la década anterior), aunque en menor grado entre los más jóvenes. Aquí se vuelven a ver los efectos de la inestabilidad y de la precariedad de los puestos de trabajo, especialmente entre los que acceden a su primer empleo.

Por último, se observan mayores diferencias entre categorías sociales en Andalucía que en Cataluña. En el primer año analizado, una distancia de cerca de 30 puntos porcentuales entre ambos extremos de la escala (jóvenes y viejos), lo que supone 10 puntos más que en Cataluña. De aquí se desprende una menor influencia de la categoría social «edad» sobre los valores en la población catalana.

Si analizamos la variable hábitat, en Andalucía se cumple la tendencia de mayor presencia de postmaterialismo en los asentamientos urbanos y de materialismo en las zonas rurales, situación que se mantiene con el paso del tiempo. Nuevamente el grado de materialismo es mayor en Andalucía; por ejemplo: en 1995 se registra un $35,3 \%$ de población materialista en las zonas rurales frente al 22,6\% presente en Cataluña ${ }^{20}$. Comparando ambas regiones, en el caso catalán apenas hay diferencias marcadas por esta categoría social, especialmente con el paso del tiempo; por ejemplo: en el 2006 hay un $1,3 \%$ de postmaterialistas puros en ambas zonas (no existen diferencias entre los extremos de la escala rural y urbana). En definitiva, el grado de postmaterialismo en Cataluña no parece estar influido por el hábitat de residencia (información que queda corroborada por el análisis del nivel de significación para el último año estudiado). De aquí se desprende un mayor grado de aleatoriedad en el comportamiento y la no influencia de esta categoría social, lo

19. De acuerdo con Inglehart (1991), según se avanza desde las cohortes más jóvenes hacia las más viejas, la proporción de materialistas sube regularmente. Por lo tanto, esta situación muestra en mayor grado un cambio generacional que efectos de edad. El hallazgo de que habría un modesto aumento del postmaterialismo simplemente a causa del reemplazo intergeneracional es consistente y se mantendrá mientras los jóvenes europeos no sean más materialistas que sus mayores, lo que ha sido confirmado por todas las investigaciones realizadas hasta el presente (Inglehart, 1991: 98). No obstante, con la caída de la tasa de natalidad desde mediados de la década de 1960, el reemplazo será mas lento y, por consiguiente, también sus efectos en los sistemas de valores.

20. Las diferencias entre Andalucía y Cataluña aparecen reflejadas más claramente si analizamos los datos de la Encuesta de calidad de vida en el trabajo, concretamente el año 1999. Para esta fecha, el porcentaje de población materialista en zonas rurales en Andalucía (33,9\%) es prácticamente el doble que en Cataluña (16,6\%). 
que vuelve a poner en evidencia un mayor grado de modernización en Cataluña.

Se demuestra una gran relación, al igual que ocurría en el caso de la edad, entre postmaterialismo y nivel educativo. En general, a menor nivel de estudios, mayor grado de materialismo, especialmente en Andalucía (por ejemplo, en 1995, la población analfabeta andaluza supera en 20 puntos porcentuales a la catalana) ${ }^{21}$. Además, las diferencias entre ambos extremos de la escala (no saber leer y universitarios) son mayores que en Cataluña. De la misma manera, a mayor formación, mayor presencia de posturas puramente postmaterialistas ${ }^{22}$. Como apunta Inglehart (2006: XI), resulta lógico que los que tienen una amplia formación o un elevado nivel de estudios tiendan a experimentar más seguridad y autonomía que otros ciudadanos de su sociedad. Con el paso de los años, como reflejan los datos de la tabla 3, se acortan las diferencias entre ambas regiones y bajan las posturas más extremas. No obstante, contrariamente a la afirmación de Inglehart, por ejemplo: población materialista con alto nivel de formación (grado medio y universitarios) — apenas existente en Cataluña en 2006-, experimenta un repunte en Andalucía: de un 17,1\% y un 14,9\%, respectivamente, en 1995 , a un $26 \%$ y un $20,3 \%$ en 2006 . Nuevamente se avista la influencia del desempleo en el mercado de trabajo andaluz, fundamentalmente de personal con un alto nivel de estudio y altamente cualificado. Esto explica que suban los niveles de inseguridad en la población y, por lo tanto, el grado de materialismo. Se vuelven a desprender diferencias por comunidades.

Otra variable que se ha tenido en cuenta en el análisis es el estatus o la posición que ocupa el individuo en la sociedad. De acuerdo con lo esperado, se explica el cambio de actitudes y de valores en función de las posiciones sociales que ocupan los individuos en la estructura social: los datos indican que a mayor posición social, mayor grado de postmaterialismo. En otras palabras, en general, se aprecia un paralelismo entre las posiciones sociales más altas y los valores postmaterialistas. Por el contrario, conforme desciende la misma, se produce un aumento de las actitudes materialistas. Esto resulta lógico si tomamos en cuenta los supuestos teóricos que apuntan que las personas con un bajo estatus están sujetas en mayor grado a necesidades de tipo económico y esto les lleva a sobrevalorar los aspectos más materialistas. De la misma manera, a medida que se avanza en la escala de estatus, se perciben incrementos regulares de dosis postmaterialistas (Bericat y Del Pino, 1998) o nuevos valores de autoexpresión asociados a una mayor seguridad personal y económica.

21. Aquí cabe recordar la tardía inversión en educación si comparamos ambas comunidades. En el caso de Andalucía, ésta no se produjo hasta bien entrado el siglo pasado.

22. Los extremos se aprecian mejor si se toman los datos de la Encuesta de calidad de vida en el trabajo. Por ejemplo, en 1999, llama la atención como el 100\% de la población en ambas regiones que no sabe leer es materialista y que, contrariamente, no se aprecia atisbo de esta tendencia entre aquellos con el nivel de estudios más elevado (tercer ciclo), concretamente en Cataluña. En esta misma línea, conforme aumenta la formación, lo hace el grado de postmaterialismo (en esta última comunidad, un $60 \%$ con estudios de tercer ciclo es postmaterialista). 
Tabla 3. Población postmaterialista según nivel educativo y posición social, según comunidad autónoma y año

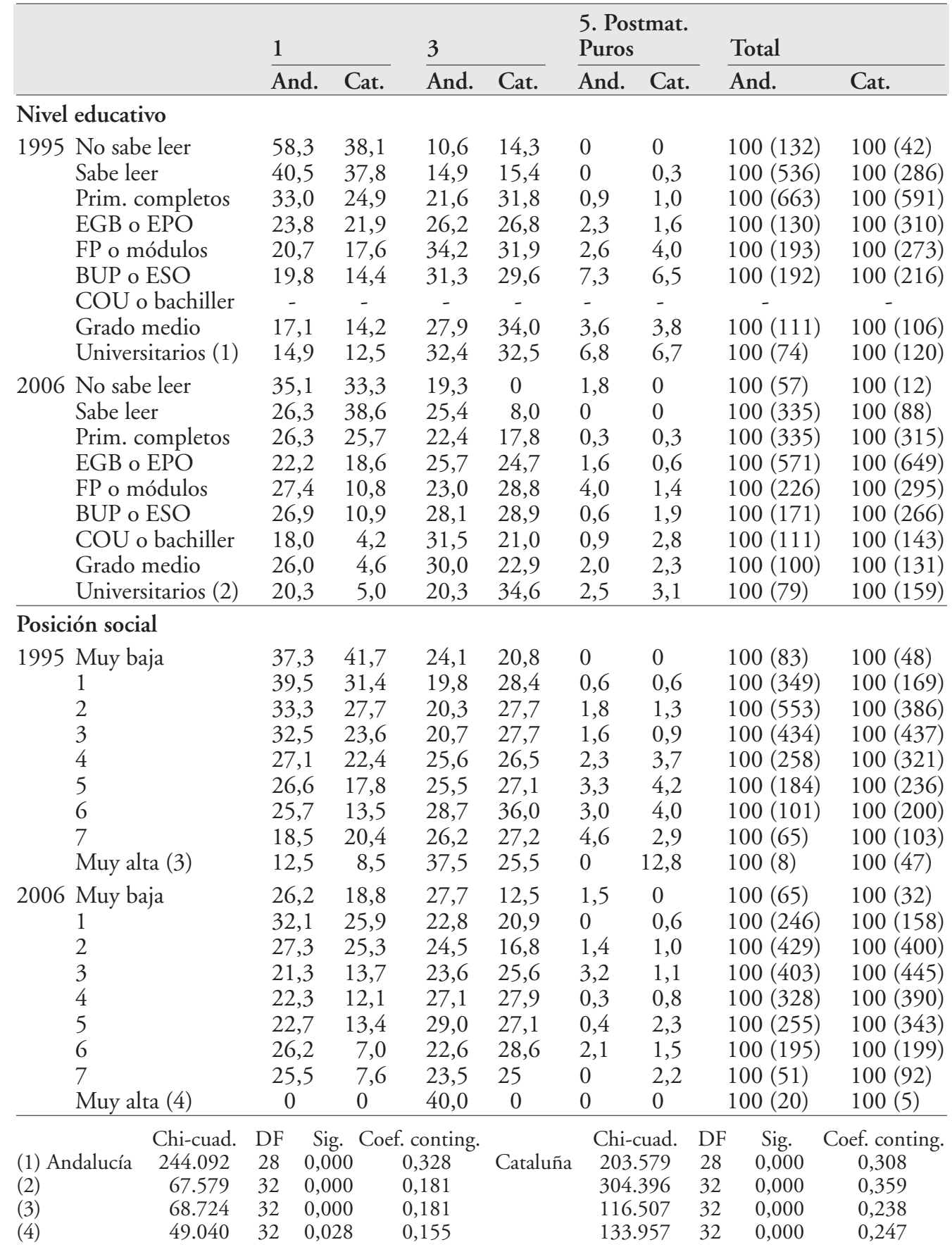

Fuente: ASEP, 1995-2006. Elaboración propia. 
Sin embargo, tras el análisis realizado, esta hipótesis no se verifica completamente en el año 2006 en Andalucía. También hay diferencias por comunidades. De hecho, tal y como se está cumpliendo hasta ahora, aparece un nuevo repunte de población materialista andaluza con alta posición social (concretamente, en los niveles 6 y 7 de la escala). En el caso de Cataluña, con el paso del tiempo, el descenso del materialismo es mucho mayor.

Pasemos a la situación laboral. Las posturas menos postmaterialistas se hallan entre aquellos con empleos temporales o inestables y, especialmente, entre los jubilados o las personas retiradas del mercado laboral. Esta situación se relaciona con la bajada del nivel de ingresos del individuo con el cese de la vida laboral, principal problema señalado en los estudios sociológicos realizados con este colectivo (véase, por ejemplo, Pérez Ortiz, 1996; Fernández Alonso, 2003, o CIRES, 1992). Por lo tanto, resulta lógico el aumento en esta etapa de valores relacionados con el crecimiento económico o el mantenimiento del orden y la seguridad. De la misma manera, posiblemente al no disponer de remuneración económica, las amas de casa también ocupan una posición relevante en este contexto.

En el extremo opuesto, los "postmaterialistas puros», como se comentaba al analizar la variable edad (mayor presencia de valores postmaterialistas en las generaciones más jóvenes), destacan los estudiantes y aquellos sujetos con puestos de trabajo de mayor nivel y prestigio. Por comunidades, con el paso de los años, se produce un descenso de la población situada en los extremos (fundamentalmente de población "materialista pura» en Cataluña). Sin embargo, en Andalucía vuelve a verse un repunte de población materialista entre aquellos sujetos que poseen una posición alta en el mercado laboral (6 puntos porcentuales más que en 1995). En el siguiente apartado se profundizará en esta cuestión.

Para terminar, ¿qué ocurre con la ideología política?23. Entre las posturas más cercanas a la derecha se da mayor grado de materialismo ${ }^{24}$ y entre las de izquierda, lógicamente en relación con elementos más puramente humanísticos, mayor grado de postmaterialismo. Más concretamente, en 1995 cerca de tres de cada diez sujetos (en ambas regiones) autodefinidos como de extrema derecha, se posicionan en el valor más bajo de la escala de postmaterialismo. Conforme se avanza en el tiempo, aumenta el materialismo en Andalucía en la extrema derecha (aunque las diferencias no son significativas estadísticamente) y en Cataluña entre los posicionados en el centro-derecha. Aquí, sorprende ver que es el único momento en el que se produce un aumento del materialismo entre la población catalana. Posiblemente ello es debido a la situación

23. Para un estudio más pormenorizado de la cultura política en España y su relación con el materialismo y el postmaterialismo, véase Jaime (2003: 204-267).

24. Esta actitud se encuentra en relación con la defensa de la ciencia altamente desarrollada y la industria como símbolo o base del progreso y la prosperidad (actitud opuesta a los postulados básicos que defiende el postmaterialismo contrarios a las grandes empresas, la ciencia establecida y los "grandes gobiernos») (Inglehart, 1991: 291). 
Tabla 4. Población postmaterialista según posición social, situación laboral e ideología política, según comunidad autónoma y año

\begin{tabular}{|c|c|c|c|c|c|c|c|c|}
\hline & \multicolumn{2}{|l|}{1} & \multicolumn{2}{|l|}{3} & \multicolumn{2}{|c|}{$\begin{array}{l}\text { 5. Postmat. } \\
\text { Puros }\end{array}$} & \multicolumn{2}{|l|}{ Total } \\
\hline & And. & Cat. & And. & Cat. & And. & Cat. & And. & Cat. \\
\hline \multicolumn{9}{|l|}{ Situación laboral } \\
\hline 1995 Alto & 13,6 & 17,7 & 31,8 & 34,2 & 9,1 & 6,3 & $100(44)$ & $100(79)$ \\
\hline Medio & 28,1 & 15,6 & 27,2 & 30,6 & 2,0 & 4,0 & $100(456)$ & $100(569)$ \\
\hline Bajo & 30,2 & 21,4 & 20,9 & 28,6 & 1,4 & 4,8 & 100 (139) & $100(84)$ \\
\hline En paro & 24,7 & 19,8 & 25,0 & 31,9 & 1,7 & 1,4 & $100(344)$ & $100(207)$ \\
\hline Ama de casa & 39,3 & 28,5 & 18,8 & 25,1 & 0,6 & 0,8 & $100(542)$ & $100(471)$ \\
\hline Jubilado & 47,2 & 35,4 & 12,2 & 22,3 & 0 & 0,3 & $100(320)$ & $100(381)$ \\
\hline Estudiante & 14,2 & 10,4 & 31,1 & 34,4 & 6,8 & 5,8 & $100(190)$ & $100(154)$ \\
\hline Otros (1) & - & - & - & - & - & - & - & - \\
\hline 2006 Alto & 19,8 & 8,5 & 26,1 & 27,7 & 1,7 & 4,3 & $100(116)$ & $100(141)$ \\
\hline Medio & 23,5 & 9,2 & 28,8 & 27,5 & 1,4 & 1,3 & $100(510)$ & $100(597)$ \\
\hline Bajo & 24,5 & 15,7 & 24,8 & 28,9 & 0,3 & 0,3 & $100(355)$ & $100(363)$ \\
\hline En paro & 28,0 & 12,5 & 22,0 & 23,1 & 0,6 & 1,9 & $100(164)$ & $100(104)$ \\
\hline Ama de casa & 27,6 & 22,8 & 23,8 & 18,9 & 0,5 & 0,3 & $100(370)$ & $100(312)$ \\
\hline Jubilado & 28,0 & 25,5 & 22,1 & 18,3 & 2,8 & 0,9 & $100(321)$ & $100(431)$ \\
\hline Estudiante & 13,7 & 3,9 & 30,2 & 26,2 & 2,9 & 3,9 & 100 (139) & $100(103)$ \\
\hline Otros (2) & 66,7 & 20,0 & 0 & 40,0 & 0 & 0 & $100(3)$ & $100(5)$ \\
\hline
\end{tabular}

\section{Ideología política}

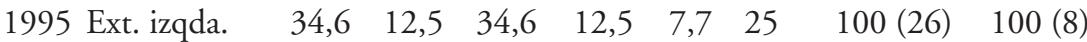

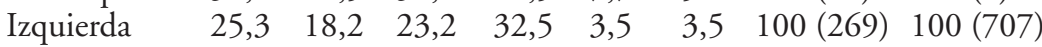

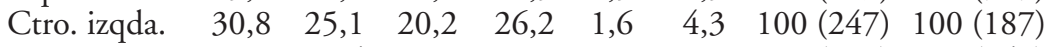

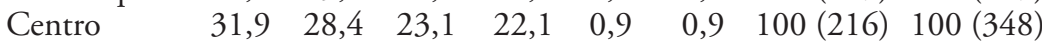

$\begin{array}{llllllll}\text { Ctro. dcha. } \quad 29,6 & 22,3 & 30,6 & 29,5 & 2,8 & 3,6 & 100(108) & 100(112)\end{array}$

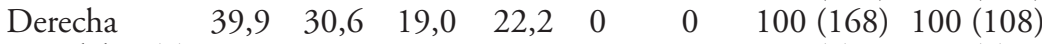

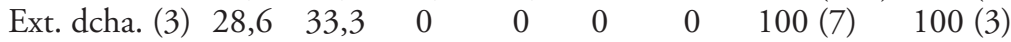

2006 Ext. izqda. $\quad 18,8 \quad 0 \quad 31,3 \quad 0 \quad 6,3 \quad 0 \quad 100(16) \quad 100(1)$

$\begin{array}{lllllllll}\text { Izquierda } & 22,5 & 4,7 & 26,2 & 31,4 & 1,9 & 1,4 & 100(676) & 100(577)\end{array}$

$\begin{array}{lllllllll}\text { Ctro. izqda. } & 19,1 & 17,0 & 31,7 & 22,4 & 1,7 & 1,6 & 100(293) & 100(745)\end{array}$

$\begin{array}{lllllllll}\text { Centro } & 20,6 & 15,0 & 29,4 & 20,7 & 0,9 & 0,8 & 100(344) & 100(353)\end{array}$

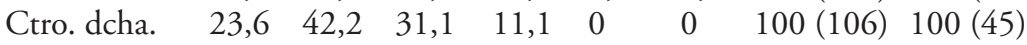

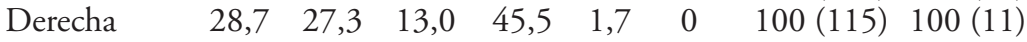

Ext. dcha. (4) $50 \quad-\quad 25,0 \quad-\quad 0 \quad 0 \quad 100(4)$

\begin{tabular}{|c|c|c|c|c|c|c|c|c|c|}
\hline & Chi-Cuad. & . DF & Sig. & Coef. Conting. & & Chi-Cuad. & I. DF & Sig. & Coef.Conting \\
\hline (1) Andalucía & 189.137 & 24 & 0,000 & 0,292 & Cataluña & 169.856 & 24 & 0,000 & 0,283 \\
\hline (2) & 61.915 & 28 & 0,000 & 0,174 & & 214.690 & 28 & 0,000 & 0,307 \\
\hline (3) & 51.903 & 24 & 0,001 & 0,189 & & 65.036 & 24 & 0,000 & 0,206 \\
\hline (4) & 36.281 & 24 & 0,052 & 0,151 & & 207.043 & 20 & 0,000 & 0,327 \\
\hline
\end{tabular}

Fuente: ASEP, 1995-2006. Elaboración propia. 
política «complicada» de los últimos tiempos (el nacionalismo, los enfrentamientos entre instituciones políticas catalanas y españolas, el nuevo Estatuto de Autonomía, la formación del Tripartito, el surgimiento de nuevas fuerzas políticas, etc.), que puede originar un aumento en las cuotas de inseguridad en ciertos sectores de la población.

Por su parte, en las posturas más postmaterialistas (avistadas en población clasificada como de extrema izquierda), despuntaba Cataluña en 1995. Sin embargo, en el último año examinado, han descendido y la distancia entre ambos extremos de la escala ideológica se acorta. Aquí es importante resaltar que la población tendente a la izquierda en el último año se posiciona mayoritariamente en el valor «2» de la escala (más cercano al materialismo). La explicación se puede encontrar en la situación política a la que se ha aludido en el párrafo anterior.

Tomando en consideración el conjunto de variables analizadas, se comprueba la existencia de una relación de la edad, del hábitat, del nivel educativo, de la posición social, de la situación laboral y de la ideología política con la escala de postmaterialismo considerada como variable dependiente ${ }^{25}$. Específicamente, la asociación más fuerte, según se desprende del análisis de los coeficientes de contingencia, se observa entre el nivel educativo y la edad con los valores postmaterialistas (presentes principalmente en jóvenes con un elevado nivel de formación). En el caso de las dos comunidades analizadas, se registran mayores dosis de postmaterialismo en Cataluña y, en general, menor influencia de las categorías sociales (mayor grado de aleatoriedad en las respuestas). Recordemos, por ejemplo, el caso de la edad (fundamentalmente en el primer año estudiado) y el del nivel educativo, donde las diferencias entre grupos o categorías sociales eran menos acusadas que en Andalucía. Por su parte, la comunidad andaluza ha destacado notablemente en mayores cuotas de materialismo, incluso produciéndose un incremento de sus niveles más extremos en determinadas categorías sociales con el transcurso del tiempo. A continuación, se verán algunas consideraciones relativas al contexto social, así como los factores que pueden estar relacionados con este proceso de cambio a lo largo del periodo de tiempo estudiado.

\section{Consideraciones finales. El postmaterialismo y los cambios sociales acaecidos en los últimos años}

Los últimos trabajos de la WVS (Díez Nicolás, 2007) permiten realizar una clasificación a nivel mundial de los países en función de su índice de postmaterialismo. En primer lugar, se encuentran aquellos que muestran una tendencia hacia un incremento del mismo: por un lado, los que reflejan un cre-

25. Estos resultados coinciden con los últimos reflejados por Díez Nicolás (2007): todas las relaciones entre postmaterialismo y edad, nivel educativo, estatus socioeconómico, posición social y muchas otras, se mantienen prácticamente en todos los países, incluso cuando las muestras tienen alguna limitación que reduce su representatividad. 
cimiento sostenido (Suecia, por ejemplo) y, por otro, una mayoría de miembros de la UE económicamente desarrollados o en vías de desarrollo rápido que siguen la tendencia de un incremento más o menos continuado de valores postmaterialistas (entre ellos, África del Sur, Alemania, Brasil, China, Dinamarca, etc.). En segundo lugar, estaría otro grupo de países (8) que reflejan una clara tendencia a la bajada de sus índices de postmaterialismo (Bielorrusia, Bulgaria, Corea del Sur, Eslovaquia, Estonia, etc.). Finalmente, otros tantos países (8) muestran un aumento de sus índices de postmaterialismo en las primeras investigaciones y un descenso importante posterior. En este caso hablamos de Argentina, Chile, España, EEUU, Francia, Italia, Japón y Países Bajos. En general, todos ellos países con un elevado desarrollo. ¿Qué es lo que ha ocurrido? ¿Por qué se produce esta caída en el nivel de postmaterialismo?

Según sostiene el autor, como se comentaba al comienzo del trabajo, en los últimos años ha aumentado la sensación de inseguridad relacionada con el surgimiento de ciertos problemas sociales que afectan a grandes sectores de la población: terrorismo, delincuencia (narcotráfico, bandas criminales internacionales, etc.) o inseguridad económica (creciente envejecimiento de la población y sus alarmantes futuros efectos en los sistemas de pensiones, en la sanidad, etc.), entre otros elementos. Esto lleva a un mayor reclamo de orden y de protección social y económica.

Extrapolando esta situación a este estudio, en Cataluña, se observa un aumento del postmaterialismo año tras año, aunque recientemente las posturas más extremas han ido descendiendo. Respecto a Andalucía, a pesar del crecimiento económico experimentado en el transcurso del tiempo ${ }^{26}$, mantiene todavía una cultura fundamentalmente materialista. Pero, ¿̨a qué se debe exactamente esta permanencia, e incluso aumento en el tiempo de valores asociados a la seguridad personal y económica y, por tanto, un descenso en los de autoexpresión? ¿Por qué no ha alcanzado todavía la sociedad andaluza un aumento significativo en sus niveles de seguridad?

Tratando de indagar en esta cuestión, aparecen en escena un conjunto de factores sociales que ayudan a responder a los interrogantes planteados. Según se desprende de los datos del último barómetro del CIS 2771 (septiembre de $2008)$, el principal problema para la población española es el paro $(62,2 \%)$ y, a continuación, los de índole económica $(54,1 \%)$ y la inmigración $(28,4 \%)$. Si descendemos al grupo social de los jóvenes (por ejemplo, entre los 18 y 24 años), la situación se repite, esta vez con un 56,9 , un 55,6 y un $32,3 \%$, respectivamente. Todos ellos están relacionados con la seguridad.

En el caso andaluz, que es el que interesa ahora, por ejemplo, en lo que a inmigración se refiere, sus costas se han convertido en una de las principales vías de acceso, fundamentalmente de marroquíes, tanto por la cercanía al continente africano, como por ser la zona mediterránea un lugar clave en el reque- 
rimiento de mano de obra barata (véase el caso de los invernaderos en la provincia de Almería). Esta situación de acrecentamiento progresivo de inmigrantes ilegales e itinerantes favorece, tal y como muestran las encuestas de opinión, el incremento de un sentimiento de inseguridad personal en la población, tanto en España como en Andalucía (por ejemplo, el estudio del CIS 2383,2000 , señala que el $51 \%$ de los españoles en ese año consideraba que la inmigración favorecía el incremento de la delincuencia en nuestro país ${ }^{27}$. En el caso andaluz, en cuanto a la percepción de efectos negativos del fenómeno migratorio, los últimos estudios mencionan la inseguridad ciudadana en un $37,1 \%$ de los casos. No obstante, aunque en anteriores encuestas se ha descrito una preponderancia relativa de las valoraciones negativas, comienzan a imponerse las positivas (Rinken y Pérez Yruela, 2007).

Por otro lado, más relevante desde mi punto de vista es la todavía inseguridad económica y social persistente en la región andaluza. En esta comunidad, como se ha comprobado a lo largo del trabajo, se le da una alta importancia a la economía, y su nivel de renta está, pese al aumento en los últimos años, muy por debajo del de Cataluña. Por lo tanto, los niveles de inseguridad e inestabilidad son mayores. Además, la precariedad y la temporalidad de los puestos de trabajo, fundamentalmente entre los jóvenes, es otra de las dificultades latentes en el territorio andaluz ${ }^{28}$. Por ejemplo, es altamente significativo que la tasa de paro en Andalucía es la más alta de España. Según datos del tercer trimestre de la EPA (2008), se sitúa en un 18,33\%. Esto es, el doble que en Cataluña (8.95) y siete puntos por encima de la del conjunto de España $(11,33 \%)$. Estos son algunos de los factores que ayudan a entender los mayores niveles de materialismo, e incluso su aumento con el paso de los años, en Andalucía. En definitiva, como diría Orizo (1996: XLV), «un materialismo propiciado por los tiempos».

\section{Conclusiones}

Los valores y las creencias de la sociedad andaluza difieren en cierta medida de los de la sociedad catalana. Tomando en consideración el conjunto de datos mostrados a lo largo del estudio, se pone de manifiesto la importancia del materialismo en Andalucía (por encima de Cataluña, en particular, y de España, en general). De manera global, se ve conformada la hipótesis de partida: la distancia entre Andalucía y Cataluña aumenta con el cambio de valores ocurrido en los últimos años.

27. Cabe recordar, especialmente en Andalucía, los violentos enfrentamientos en El Ejido (Almería) entre autóctonos e inmigrantes que han ocupado en numerosas ocasiones las portadas de periódicos en los últimos años.

28. Como señalan del Pino y Bericat (1998: 122): «por ejemplo, un 46,1\% de andaluces se decantan por "un trabajo seguro que no comporte riesgos de cierre o desempleo" y sólo un 22,7 demanda un "buen salario". La opción de seguridad es mayor en Andalucía que en España (40,3\%) y la importancia de un buen sueldo es menor en Andalucía que en España». 
En relación con las distintas categorías sociales, se ha comprobado: 1) la existencia de una relación directa entre la edad y el nivel educativo con los valores postmaterialistas, es decir, los valores postmodernos, de autoexpresión o emancipación están más presentes en los estratos más jóvenes y con mejor formación; 2) aunque en menor proporción, también ocurre con el hábitat, la posición social, la situación laboral y la ideología política, esta vez predominan los valores postmaterialistas en las zonas urbanas y entre los sujetos con más recursos, mayor posición social, estabilidad laboral ${ }^{29}$ y tendentes a la izquierda; 3) no obstante, no siempre se cumplen estas tendencias (recuérdese, por ejemplo, el caso de población materialista en el último año analizado: joven, con alta posición social y nivel educativo elevado en Andalucía), puesto que se aprecia la influencia de otros factores sociales (niveles de renta, desempleo, etc.); 4) la influencia de las categorías sociales es mayor en Andalucía que en Cataluña, donde se reconoce una mayor aleatoriedad en el comportamiento de la población (por ejemplo, claramente se veía en el caso del hábitat: actualmente, esta variable no ejerce influencia en el grado de postmaterialismo de la población catalana, donde se registra el mismo número de postmaterialistas en las zonas rurales y urbanas). En las sociedades con mayor grado de modernización, se cumple, como se señalaba en el marco teórico, la manifestación de cierta independencia del comportamiento individual con respecto a las categorías sociales.

Aunque se observan cambios en los años más recientes favorables hacia actitudes más postmaterialistas (autonomía, creatividad y autoexpresión), pero no en el valor más extremo de la escala, que ponen de manifiesto mayores niveles de modernización, fundamentalmente en Cataluña, otra cuestión importante es el repunte, esta vez en Andalucía, de valores materialistas. Fundamentalmente aquellos relacionados con la estabilidad económica y con la seguridad. Así mismo, se produce una bajada en aquellos de corte postmaterialista o relacionados con la autoexpresión (participación social e importancia de las ideas).

En este último sentido, se cumple una de las hipótesis señaladas por Inglehart y Welzel (2006) en la actualidad: una bajada en la seguridad personal y económica de la población que implica una disminución en los «valores de autoexpresión». De manera contraria, cuando las poblaciones adquieren mayores grados de seguridad personal y económica, los individuos van abandonando su preocupación por los "valores de escasez o supervivencia» y se orientan hacia otros nuevos «valores de autoexpresión o de emancipación». Como se ha visto, hoy en día muchos países, entre ellos el nuestro, están experimentando recortes en su seguridad personal, por lo que resulta lógica la mencionada bajada de los «valores de autoexpresión o emancipación» y la subida de los «valores de escasez o supervivencia» (Díez Nicolás, 2006).

29. Cabe recordar que, rompiendo esta tendencia, al analizar la situación laboral también aparecen los estudiantes como uno de los colectivos con mayor presencia de valores postmodernos. No obstante, esto resulta lógico si recordamos lo que ocurría con el caso de la edad (mayor grado de postmaterialismo entre los más jóvenes). 
En el caso de Andalucía, se aprecia claramente esta tendencia. Se constata que aún siguen afectando considerablemente determinados factores, como los económicos, en los valores aquí estudiados. A su vez, se comprueba que Cataluña, con el paso del tiempo, se va alejando más del peso o la influencia de este tipo de variables. En definitiva, aún caminando ambas poblaciones hacia la postmodernidad, Cataluña tiende a hacerlo a un ritmo más rápido que Andalucía.

\section{Referencias bibliográficas}

BELL, D. (1973). The coming of post-industrial society: A venture in social forecasting. Nueva York: Basic Books, Inc.

Bericat, E. (2003). «Fragmentos de la realidad social posmoderna». Revista de Investigaciones Sociológicas, 102, abril-junio, 9-45.

CARnero, T. (1992). Modernización, desarrollo politico y cambio social. Madrid: Alianza Universidad.

CIRES (1992). Tercera edad. Madrid: Centro de Investigaciones sobre la realidad social. Boletín mes de mayo.

CIS (2000). Estudio 2383. Madrid: Centro de Investigaciones Sociológicas. Barómetro de septiembre.

- (2008). Estudio 2771. Madrid: Centro de Investigaciones Sociológicas. Barómetro de septiembre.

COMAS, D. (coord.) (2003). Jóvenes y estilos de vida: Valores y riesgos en los jóvenes urbanos. Madrid: FAD, INJUVE.

Díez Nicolás, J. (2006). «Prólogo». En: Inglehart, R. y Welzel, Ch. Modernización, cambio cultural y democracia: La secuencia del desarrollo humano. Madrid: CIS, IX-XIII.

- (2007). «¿Regreso a los valores materialistas?: El dilema entre seguridad y libertad en los países desarrollados». IX Congreso Español de Sociología "Poder, cultura y civilización». Barcelona, 13-15 de septiembre.

EISENSTADT, S.N. (1964). «Breakdowns of modernization». Economic Development and Cultural Change, 25, suplemento, 67-98.

EPA (2008). Tercer trimestre 2008. Madrid: Instituto Nacional de Estadística.

FERNÁNDEZ Alonso, M. (2003). El proceso de jubilación y sus consecuencias. Granada: Facultad de Ciencias Políticas y Sociología. Documentos de Trabajo; 20.

GOBERNADO, R (coord.) (1996). Análisis comparado de las estructuras sociales de Andalucía y Cataluña. Universidad de Málaga y Universidad de Almería.

- (2004). «La cultura arbitraria. Operativización del concepto». Revista Internacional de Sociología (RIS). Tercera época, 37, enero-abril, 29-46.

INGLEHART, R. (1991). El cambio cultural en las sociedades industriales avanzadas. Madrid: CIS. Monografías; 121.

INGLEHART, R. y WeLZeL, Ch. (2006). Modernización, cambio cultural y democracia: La secuencia del desarrollo humano. Madrid: CIS. Monografías; 231.

JAime, A.M. (2003). «La cultura política en España: Entre el materialismo y el postmaterialismo». En: BERICAT, E. (dir.). El conflicto cultural en España: Acuerdos y desacuerdos entre los españoles. Madrid: CIS, 204-267. Monografías; 202.

MARX, K. (1986). El capital. Barcelona: Orbis.

OrIzO, F.A. (1996). Sistemas de valores en la España de los 90. Madrid: CIS. Monografías; 150. 
PINO, J. del y BERICAT, E. (1998). Valores sociales en la cultura andaluza: Encuesta mundial de valores. Andalucía 1996. Madrid: CIS. Monografías; 156.

REQUENA, F. (2002). "Cambio de valores y desarrollo». En: La sociedad: teoría e investigación empirica. Libro homenaje a José Jiménez Blanco. Madrid: CIS, 1027-1049.

- (2004). «Modernización y postmodernización: Elementos para una teoría reflexiva del cambio en las estructuras sociales». En: Reflexiones sociológicas. Libro homenaje a José Castillo Castillo. Madrid: CIS, 323-343.

Rinken, S. y PÉrez Yruela, M. (2007). Opiniones y actitudes de la población andaluza ante la inmigración. Sevilla: Consejería de Gobernación. Junta de Andalucía. Estudios y Monografías; 5.

Solé, C. (1976). Modernización: un análisis. Barcelona: Península.

SzTOMPKA, P. (1995). Sociología del cambio social. Madrid: Alianza.

Toffler, A. (1980). The third wave. Nueva York: Bantam Books.

TURNER, J.H. (1971). "Patterns of value change during economic development: An empirical study». Human Organization, 30 (2), 126-136.

WeBER, M. (1979). La ética protestante y el espiritu del capitalismo. Barcelona: Península.

Welzel, Ch.; InglehaRT, R. y Klingemann, H.-D. (2003). "The theory of human development: A cross-cultural analysis». European Journal of Political Research, 42, 341-379. 